\title{
To Compare the Effectiveness of Transurethral Pneumatic Lithotripsy and Extracorporeal Shock Wave Lithotripsy: In treatment of Lower Ureteric Stones
}

\author{
Siddique Ahmad', Jamshed', Ameroon Shah², Riaz Ahmad Khan², Muhammad Kalim¹, Sohaib Ali
}

\begin{abstract}
Background: One of the most common complaint in the ER is colic due to Ureteric calculi. They usually pass out but stones $>5 \mathrm{~mm}$ may require intervention. ESWL and TPL are one of the treatment options.

Objectives: To compare the effectiveness of transurethral pneumatic lithotripsy (TPL) and extracorporeal shock wave lithotripsy(ESWL) in treatment of lower ureteric stones.

Material and Methods: Over period of six months a group of 70 patients presenting with ureteric calculi were randomly allocated in two groups. Group Aunderwent TPL while Group B ESWL. Follow up was done to determine the effectiveness with regards to stone clearance.

Results: The mean age of the whole study sample was $35.6+8.5$ years. The difference between mean age of both groups was statistically not significant ( $p$ 0.665). there were $70 \%$ males compared to $30 \%$ females in the overall sample and the difference of gender across both groups was comparable ( $p$ 0.192). The mean size of stone of the whole study sample at presentation was $6.4+$ $2.5 \mathrm{~mm}$ and the duration of illness was $4.8+1.8 \mathrm{months}$. On follow up, the effectiveness of the procedure in terms of stone clearance
\end{abstract} was recorded in $88.6 \%$ of TPL group and $77.1 \%$ of ESWL group ( $p$ 0.205).

Conclusion: TPL is comparable to the ESWL in terms of its effectiveness for ureteric calculi of size less than or equal to $10 \mathrm{~mm}$. More research trials need to be done for conclusive outcome.

Key Words: Urolithiasis, ureteric calculi, transurethral pneumatic lithotripsy, extracorporeal shock wave lithotripsy, stone clearance.

This article may be cited as: Ahmad S, Jamshed, Shah A, Khan RA, Kalim M, Ali S. To Compare the Effectiveness of Transurethral Pneumatic Lithotripsy and Extracorporeal Shock Wave Lithotripsy: In treatment of Lower Ureteric Stones. J Saidu Med Coll Swat. 2021;11(4):223-9.DOI:https://doi.org/10.52206/jsmc.2021.11.4.683

\section{INTRODUCTION}

To Compare the Effectiveness of Transurethral Pneumatic Lithotripsy and Extracorporeal Shock Wave Lithotripsy: In treatment of Lower Ureteric Stones.

One of the most common complaint in the ER is colic due to Ureteric calculi ${ }^{1}$. Although common it affects men more (12\%) compared to women (6\%) and most of them are in their prime when they get it. Ureteric stones usually present with severe pain, colicky in nature, radiating down, burning micturition, with dark urine and sometimes nausea, vomiting if very severe requiring opioids to be administered. They are usually treated with fluids, antispasmodics and pain killers, in case of treatment failure, ESWL (extracorporeal shock wave lithotripsy) and TPL (transurethral pneumatic lithotripsy) are the preferred options. They usually pass on spontaneously but multiple factors related to the local anatomy, stone size, associated spasm, hydration status and infection, all can affect whether or not it will pass on without any help, its commonly noted that stones $>5 \mathrm{~mm}$ will usually require some form of intervention ${ }^{2,3}$.

1. Department of General Surgery, Hayatabad Medical Complex

2. Department of Urology, Institute of Kidneys Disease Hayatabad

Correspondence: Dr. Syed Ameroon Shah

Medical officer, Department of Urology,IKD

Email: shahameroon@gmail.com
ESWL uses focused sonic energy applied from the outside the body flush against the skin to break up the stone by causing vibrations within its internal structure, its easy, its safe, and no need to give any pain medication or anesthesia. As a procedure ESWL is quite straight forward and can be performed with minimum fuss in outpatient. In contrast, transurethral pneumatic lithotripsy requires aseptic measures as a ureteroscope is guided up the urethra into bladder and then up to the ureter, then a pneumatic lithotripter device goes up the scope and fragments the stones at the level of obstruction, this procedure is minimal invasive and is a surgical procedure and entails with it all the requisite surgical complications ${ }^{4}$.

Razzaghi et al, in their study comparing pneumatic lithotripters versus holmium laser for ureteral calculi, concluded that immediate stonefree rate in pneumatic lithotripsy group was $82.1 \%$ while in $17.9 \%$ patients stone was pushed backward. Three of them were managed with conservative measures while the remaining patients needed extracorporeal shock wave lithotripsy. Mean duration of pneumatic lithotripsy was $7.9+4.2$ minutes for this group. Mucosal damage \& postoperative fever occurred in $1.8 \%$ $\$ 3.6 \%$ of patients' respectively ${ }^{5}$. A study on the comparison of efficacies of holmium YAG laser, and pneumatic lithotripsy in the endoscopic treatment of ureteral showed that transurethral pneumatic lithotripsy has $98.4 \%$ stones-free rate 
for distal ureter. The operation time was significantly shorter with less postoperative analgesics requirement $\&$ shorter hospital stay for transurethral pneumatic lithotripsy ${ }^{6}$. In a prospective study for management of distal ureteric stone Etafy M et al. noted that in the ESWL group, the operative time was $43.13+22.5 \mathrm{~min}$; the average number of sessions/patients was 1.5 sessions; the average number of SW/patients was $4500 \mathrm{SW} /$ patients; the average energy was 16.5 $\mathrm{kV}$; the average stone burden was $7.8 / \mathrm{mm}$; the overall stonefree rate was $75 \%(36 / 48)$; and the average radiation exposure time was $3.5 \mathrm{~min}^{7}$. A prospective randomized study by Verze $\mathrm{P}$ et al. showed that stone-free rate for distal ureteric stones treated with ESWL was $92.70 \%$ with a $44.88 \%$ re-treatment rate and an $11.02 \%$ auxiliary procedure rate ${ }^{8}$. The rationale of the current study is to know about the effectiveness of transurethral pneumatic lithotripsy and extracorporeal shock wave lithotripsy in treatment of lower ureteric stones cases in our population because there is not any recent research available in local adult population as very few studies in literature tackle this issue andif they did, most of them were retrospective analysis, this warranted a prospective study based on local data. The current study will contribute to serve as a platform in devising local guidelines for adopting one technique in preference to the other, affecting patient management. The study will lead us towards the better management of patients with lower ureteric stone cases.

\section{MATERIALAND METHODS}

This study was conducted in Department of General Surgery and Department of Urology, Institute of Kidney Diseases, Hayatabad, Peshawar, from July 2018, to January 2019, a total duration of 06 months. Study design was randomized controlled trial. Total number of patients was 70,35 in each group. Keeping a stone free rate in pneumatic lithotripsy as $98.4 \%$ and $75 \%$ respectively as a success rate in ESWL based on previous study.6,7 significance level was $5 \%$ and power was $90 \%$ under WHO sample size calculation formula.

\section{Sampling Technique}

Consecutive non-probability sampling Inclusion criteria
1. Both genders
2. Age 18 to 50
3. Patients with single lower ureteric stone of $10 \mathrm{~mm}$ or less in size being unilateral or

bilateral as evident on radiographs or ultrasound \& with symptoms of flank pain radiating to genitalia for at least 2 weeks.

\section{Exclusion Criteria}

1. Patients with solitary kidney as evident on ultrasonography.

2. Renal insufficiency i.e. serum creatinine $>=3 \mathrm{mg} / \mathrm{dl}$.

3. IVU showing a stricture in the ureter one side only.

4. UTI on urine examination.

5. Transplanted kidney or previous surgery for ureteric stones known from patient history \& examination.

6. Morbid obesity or pregnancy confirmed on ultrasound.

7. Bleeding diathesis as evident on clotting profile.

\section{Data Collection Procedure}

All the patients coming to out-patient department, diagnosed as having lower ureteric calculi \& meeting the selection criteria, was included in the study. They were randomly distributed into two groups ( $A$ \& $B$ ) each group having equal number of patients. A written consent both for the procedure \& inclusion in the study was taken, followed by detailed history and thorough examination. Ultrasound followed by a KUB x-ray and for tract assessment an IVU would be done and examined by a consultant radiologist having at least 2 years of experience, for the diagnosis of ureteric stone. TPL treatment group was A, while ESWL (group $B)$. TPL required the $A$ group to be admitted day before, while the $B(E S W L)$ group was treated as outpatient. All the patients were given intravenous a single dose of diclofenac sodium $50 \mathrm{mg}$ post procedure for pain control in both groups. All patients were given prophylactic oral ciprofloxacin $500 \mathrm{mg}$ twice a day for 5 days. At each visit post procedure fluoroscopy informed upon the disease free status of the both groups. Presence of no stone in ureter on fluoroscopy was termed as treatment success (100\% stone clearance). Following the procedures, complete stone clearance was further confirmed by X-ray KUB \& ultrasound which was reported by the same consultant radiologist. A pre-designed proforma was used for data collection.

\section{Statistical Analysis}

All data was analyzed using SPSS version 20. All variables like age, gender, duration of illness, stones size, stone number and efficacy of 
intervention was computed. Chi-square test was used to compare the success of therapeutic intervention in both the groups. A $P$ value of less than 0.05 was considered significant. Effectiveness is stratified among age, gender and duration of illness to see the effect modifiers using chi square test with $\mathrm{P}$-value $<0.05$ was considered significant. All the results are presented in the form of tables and charts.

\section{RESULTS}

The study was conducted on 70 patients urolithiasis of size less than $10 \mathrm{~mm}$. All patients were randomly allocated in two groups. Patients in group A were subjected to tranuretheral pneumatic lithotripsy (TPL) while patients in group $B$ were subjected to extracorporeal shock wave lithotripsy (ESWL).

The mean age of the whole study sample was 35.6 \pm 8.5 years. Minimum age of 21 years and maximum age of 50 years. The difference between mean age of both groups was statistically not significant ( $p$ 0.665).

While distributing the patients with regards to gender, there were $70 \%$ males compared to $30 \%$ females in the overall sample. The difference of gender between both groups was statistically not significant ( $p 0.192)$.

The mean size of stone of the whole study sample at presentation was $6.4 \pm 2.5 \mathrm{~mm}$. We categorized the stones between $1-5 \mathrm{~mm}$ and $>5-10 \mathrm{~mm}$ and compared between both groups which was statistically not significant ( $p$ 0.212). (Graph 01)

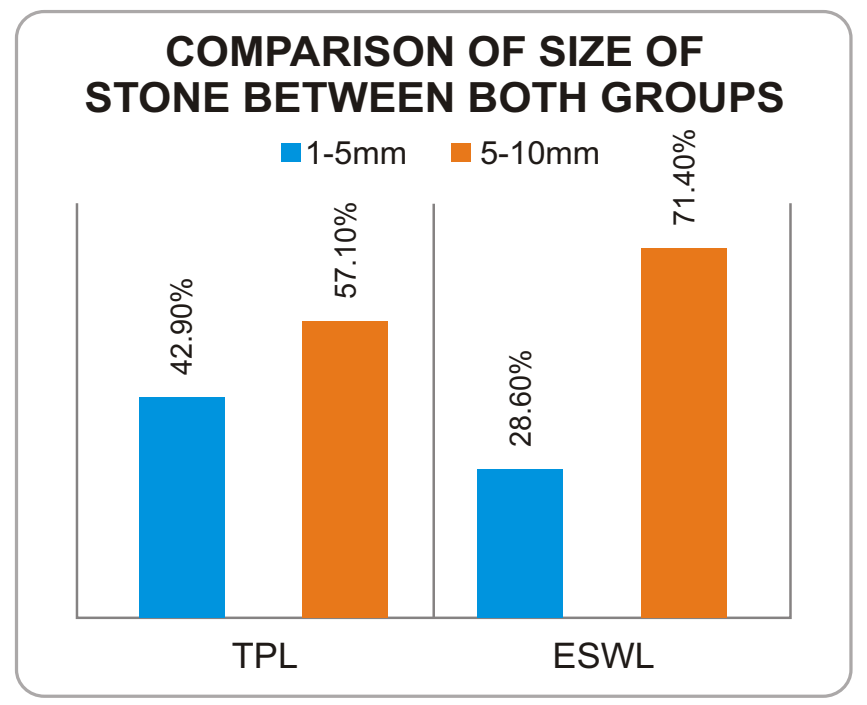

Graph 01: Comparison of stone size between both groups
The mean duration of illness of the whole study sample was $4.8 \pm 1.8$ months. we categorized the duration of illness as 2-5 months and $>5-8$ months and compared between both groups ( $p 1.0)$.

On follow up, the effectiveness of the procedure in terms of stone clearance was recorded in $88.6 \%$ of TPL group and $77.1 \%$ of ESWL group ( $p 0.205$ ). (Table1)

Table 1.

Comparison Of Effectivenss Of Treatment Between Both Groups ( $n=35$ Each)

\begin{tabular}{|c|c|c|c|c|}
\hline & \multicolumn{2}{|c|}{ Treatment Groups } & \multirow{2}{*}{$\begin{array}{c}\text { P } \\
\text { VALUE }\end{array}$} \\
\hline & & TPL & ESWL & \\
\hline \multirow{4}{*}{$\begin{array}{c}\text { Effectiveness } \\
\text { of treatment }\end{array}$} & \multirow{2}{*}{ Yes } & 31 & 27 & \multirow{6}{*}{0.205} \\
\hline & & $88.6 \%$ & $77.1 \%$ & \\
\hline & \multirow{2}{*}{ No } & 4 & 8 & \\
\hline & & $11.4 \%$ & $22.9 \%$ & \\
\hline \multirow{2}{*}{\multicolumn{2}{|c|}{ Tota I }} & 35 & 35 & \\
\hline & & $100.0 \%$ & $100.0 \%$ & \\
\hline
\end{tabular}

In our study, age wise distribution showed treatment effectiveness of $100 \%$ for ESWL in age group 20-30 years while TPL showed a higher success rate in patients aged 30 years and above of $83.3 \%$ compared to $63.6 \%$ failure of ESWL Gender wise, females had a higher success rate with TPL( $84.6 \%$ ) compared to a failure rate of ESWL (62.5\%) while in male gender, TPL and ESWL had comparable $90.9 \%$ \& $88.9 \%$ success rate respectively. In terms of stone size $(5 \mathrm{~mm})$, TPL had a $100 \%$ treatment effectiveness compared to ESWL (70\%) while stone sized 5$10 \mathrm{~mm}$, TPL and ESWL had equal $80 \%$ treatment effectiveness (Table 2-3).

Table 2.

Baseline Size Of Stone 1-5mm Wise Stratification Of Effectiveness

\begin{tabular}{|c|c|c|c|c|}
\hline & \multicolumn{2}{|c|}{ Treatment Groups } & \multirow{2}{*}{$\begin{array}{c}\text { P } \\
\text { VALUE }\end{array}$} \\
\hline & & TPL & ESWL & \\
\hline \multirow{4}{*}{$\begin{array}{c}\text { Effectiveness } \\
\text { of treatment }\end{array}$} & \multirow{2}{*}{ Yes } & 15 & 7 & \multirow{6}{*}{0.024} \\
\hline & & $100.0 \%$ & $70.0 \%$ & \\
\hline & \multirow{2}{*}{ No } & 0 & 3 & \\
\hline & & $0.0 \%$ & $30.0 \%$ & \\
\hline \multirow{2}{*}{\multicolumn{2}{|c|}{ Tota I }} & 15 & 10 & \\
\hline & & $100.0 \%$ & $100.0 \%$ & \\
\hline
\end{tabular}


To Compare the Effectiveness of Transurethral Pneumatic Lithotripsy...

Table 3. Baseline Size Of Stone > 5-10 mm Wise Stratification Of Effectiveness

\begin{tabular}{|c|c|c|c|c|}
\hline & & \multicolumn{2}{|c|}{ Treatment Groups } & \multirow{2}{*}{$\begin{array}{c}\mathbf{P} \\
\text { VALUE }\end{array}$} \\
\hline & & TPL & ESWL & \\
\hline \multirow{4}{*}{ Effectiveness of treatment } & \multirow{2}{*}{ Yes } & 16 & 20 & \multirow{6}{*}{1.0} \\
\hline & & $80.0 \%$ & $80.0 \%$ & \\
\hline & \multirow{2}{*}{ No } & 4 & 5 & \\
\hline & & $20.0 \%$ & $20.0 \%$ & \\
\hline \multirow{2}{*}{\multicolumn{2}{|c|}{ Total }} & 20 & 25 & \\
\hline & & $100.0 \%$ & $100.0 \%$ & \\
\hline
\end{tabular}

Treatment effectiveness of both procedures was assessed by total duration of illness which show that in a shorter follow up of 2-3 months, TPL had treatment effectiveness of $81.8 \%$ compared to ESWL(77.3\%) but when the patients were followed up to 5-8 months, TPL had a success rate of $100 \%$ while in the ESWL, $76.9 \%$ were successfully treated with a $23 \%$ failure rate Graph 02.

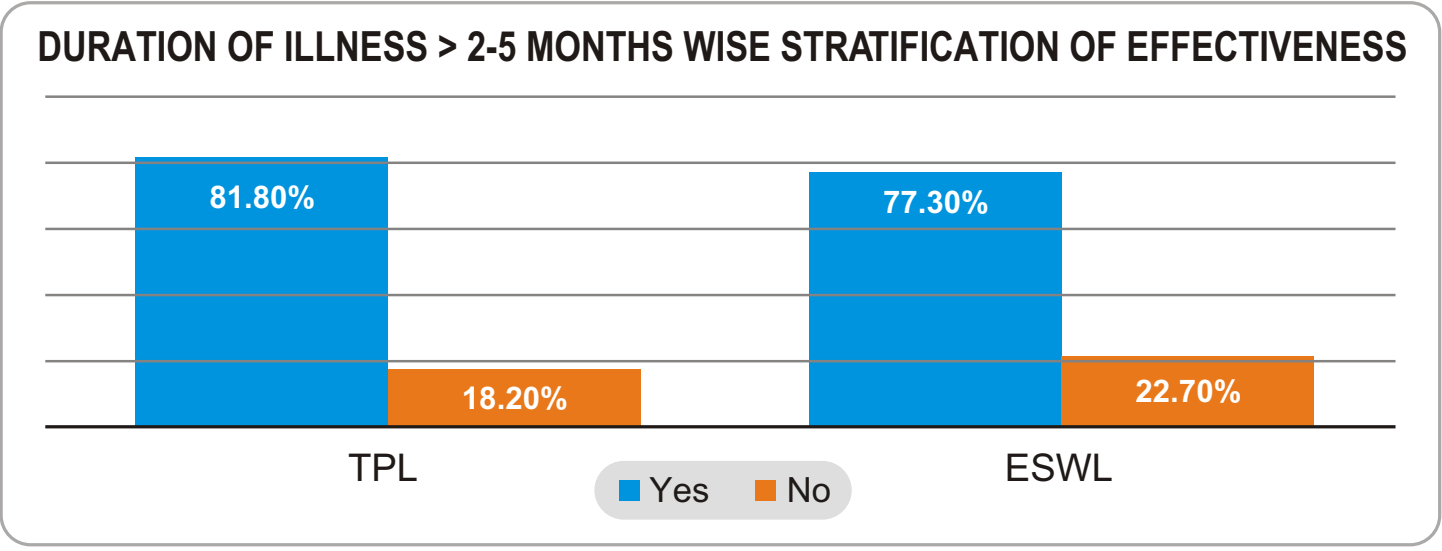

\section{Graph 02: Duration of illness > 2-5 months wise Stratification of Effectiveness of Treatment}

\section{DISCUSSION}

Spontaneous clearance of stones is common enough for ureteric stones, and time should be given for the stones to pass on by itself ${ }^{9,10}$ AnAUA met analysis found that almost all stones less than $5 \mathrm{~mm}$ will pass in $98 \%$ of case subjects, though also dependent upon the locally anatomical factors also nonetheless. ${ }^{9}$ It is imperative for the attending physician to wait for an appropriate amount of time 4-6 weeks in ureteric stones with obstructive features, and only intervene if the complications are present. ${ }^{11,12}$ As in all cases timing is the key and ensures success ${ }^{13}$ In retrospect if anyone looks at the data of the spontaneous clearance there is a clear connection for the size of stone and spontaneous clearance, so it is prudent to wait a while especially in small stones, so that a therapeutic intervention is better evaluated against other interventions in terms of efficacy ${ }^{13}$ In our study, patient aged 30 and above had almost $80 \%$ success rate compared to ESWL with a failure rate of $63.6 \%$ in the specific age group. A recent study conducted by Peschel et al states that there are clear differences between ESWL and TPL, specifically the TPL is superior in that it's shorter procedure and a stone free status is achieved in rather shorter amount of time compared to ESWL and the study further recommended the TPL as a first line treatment option for stones less than $5 \mathrm{~mm}$ not passing spontaneously.

Compared with distal ureteral stones, TPL performed for stones in the proximal ureter have been associated with lower success rates which are ascribed to a more difficult access as well as the proximal migration of stone fragments in TPL. In some studies it was found that compared with the ESWL group treatment cost was higher in the TPL group due to hospitalization and inpatient 
costs. ${ }^{14}$ The best modality for the treatment of ureteral stones is still debatable. ${ }^{14}$ Patients who stay far from hospital more probably choose TPL over ESWL to avoid frequent visits because of its high success rate for single treatment. ${ }^{15}$ Patient's economic status also effects satisfaction level of the patients regarding choice of TPL or ESWL for treating their ureteral stones especially in a society like Pakistan where people with middle, and low-income levels have difficulty in coping with the costs incurred in private setups. ${ }^{15}$ In literature there is a controversy regarding the financial burden on patients. ${ }^{16}$

There are some important factors such as stone location, size, composition, surgeon's inclination and patient's choices that play a vital role in the decision regarding the use of open, laparoscopic, ESWL or TPL for treating ureteral stones. In modern day practice, SWL and TPL are considered as the first-line treatment modalities for the treating ureteral stones. ${ }^{17}$

Shock wave lithotripsy is a noninvasive procedure, that do not require much hassel, however it has its own disadvantages as long treatment time, high retreatment rate and poor compliance by the patient. ${ }^{15}$ AUA recommendations indicated that ESWL be used as the first-line of management option for a small stone $(<1 \mathrm{~cm})$ but indications had not been so clear for using it for proximal ureteral stones of more than $1 \mathrm{~cm}^{18,19}$ The advancement in technology has led to introduction of small caliber semi-rigid ureteroscope. A combination of TPL and intracorporeal lithotripsy has proven to be a viable alternative to ESWL. ${ }^{18}$ In our study, we found that TPL had a shortened duration of illness with treatment success in $80 \%$ of patients while ESWL had a treatment effectiveness of $77.3 \%$ in a 2-3 months follow-up but when compared a longer follow-up of 5-8 months, the treatment TPL was effective in $100 \%$ cases while ESWL had a failure rate of $23.1 \%$.

In a study done in Pakistan it was concluded that ESWL was the preferred choice of treatment for proximal ureteral stones, but ureterorenoscopic manipulation with intracorporeal lithotripsy was also safe for a quicker relief of symptoms in patients with proximal ureteral stones. ${ }^{20}$ Laparoscopic approaches are reasonable alternatives in cases, where ESWL and TPL have failed. However, they did not mention the economic burden of these procedures on the patients.

In our study, we found that based on stone size 1$5 \mathrm{~mm}$, TPL had a $100 \%$ success rate while ESWL had $30 \%$ failure rate and then in the stone size of $5-10 \mathrm{~mm}$, TPL and ESWL both had comparable $80 \%$ success rate respectively. The success rate (stone free rate) of TPL has been around $80 \%$ in the proximal ureter. It is seen in literature that TPL has a higher stone-free rate for stones smaller than or equal to $10 \mathrm{~mm}$ in the distal ureter and stones bigger larger than $10 \mathrm{~mm}$ in the proximal ureter. $^{21}$ It is pertinent here that besides the influence of stone size and position, the efficiency of the TPL procedure depends on the experience of the TPL procedure depends on the experience and skill of the operating urologist as well. ${ }^{22}$

Besides thinking about all the factors while deciding a specific treatment modality, priority should be always given to patient's preference. Some patients might have concerns regarding the anesthesia requirement and the invasive nature of TPL. But there may be other set of patients who might prefer to have their stone removed and the pain alleviated at the earliest possible time, thus avoiding multiple treatment sessions that might be required in case of SWL as treatment modality. ${ }^{23}$

Though another study which generated a high success rate with TPL notes that the added cost is more complication and longer hospital stay. ${ }^{24}$ However during the course of our study, we have had more complications associated with TPL subgroup with nominal hospital stay of 1 day post procedure. Lee et al. noted no major differences regarding patients' satisfaction for either intervention (TPL or ESWL). ${ }^{25}$ A cost benefit study carried out in Egypt noted that ESWL was on average 700 EGP cheaper compared to TPL.(24) Lee et al. also noted the same in USA with ESWL being cheaper by 517 dollars from TPL. ${ }^{25}$ Strangely enough the TPL becomes a cheaper option compared to ESWL if stones are larger than $10 \mathrm{~mm}$, the author attributes this to the number of sessions required for ESWL to be effective is much more. ${ }^{26}$

All interventions failure and success has only one denominator, stone size. ${ }^{27}$ One another factor in the context of ESWL for ureteral stones treatment is the skin to stone distance, which has also a role in determining stone-free rates. ${ }^{27}$ Significantly in the age group 20-30 years, the success rate of 
ESWL was $100 \%$ in our study which hints at the significance of skin to stone distance in the context of ESWL procedure due to the observed fact that younger people have more lean physiques (nominal BMI). A recent analysis of the use of the CT Scan to predict beforehand whether or not the ESWL treatment would be efficacious based on the predication of stone composition, did not yield conclusive outcomes. Further research needs to be conducted to ascertain that. ${ }^{28,29}$ Massoud et al. stated that in patients who have stones of 500 to $1000 \mathrm{HU}$, factors such as body mass index of more than $30 \mathrm{~kg} / \mathrm{m}^{2}$ and a lower calyceal location make them less suitable for ESWL. ${ }^{29}$ So the studies done previously have not accounted for these confounding factors while comparing the TPL and ESWL modality for the proximal ureter stones.

Alameddine et al. reported the complications of TPL including perforation in $5 / 103(4.8 \%)$ patients which were treated with double-J stents except one patient who required nephrostomy tube placement and a laparotomy for intraperitoneal drainage of collection. ${ }^{31}$ Two patients $(1.94 \%)$ developed sepsis. Their initial stone-free rate for proximal TPL Group was $89 \%$. Their mean stone size was $10 \mathrm{~mm} \pm 5.5 \mathrm{~mm}$ which was smaller. In another study stone- free rate after TPL was $86.7 \% .^{31}$ In a study by Al-Marhoon et al, in ESWL of ureteral stones, the majority of the complications were minor with the most common being the loin pain in $21 \%$ of the patients. ${ }^{32}$ In a study by Salem et al. complications in the TPL group included 4 cases $(4 \%)$ having mild extravasation which were managed by DJ stenting. ${ }^{24}$ Aboutaleb et al. reported complications including steinstrasse in $34.8 \%$ (ESWL group) and 3.7\% (TPL group) of the patients, and ureter perforation in $0 \%$ (ESWL) and $7.4 \%$ (TPL) of the patients. ${ }^{25}$ While we had ureteral perforation in 2 cases ( $1 \%)$.

There is marked heterogeneity of evidence in different papers. The size of stone, whether a person is morbidly obese, the number of stones, how often the physician performs the procedure, technologies used, when all of this is taken into account then we can say which intervention is best for the patient. Although a landmark study in our setup,however, we did not take into account the different factors that may affect stone free rates in ESWL or TPL groups while comparing the stone clearance rates for TPL or ESWL for ureteral stones. Our results need to be investigated more in larger groups and multicenter prospective studies

\section{CONCLUSION}

TPL is comparable to the ESWL in terms of its effectiveness for ureteric calculi of size less than or equal to $10 \mathrm{~mm}$. TPL can be recommended for quick relief of symptoms but the procedure should be performed in the center of excellence by the competent hands. In Pakistan, ESWL is the cost effective treatment of choice and can be ideal for people with nominal BMls.

\section{REFERNCES}

1. Fwu CW, Eggers PW, Kimmel PL, Kusek JW, Kirkali Z. Emergency department visits. use of imaging, and drugs for urolithiasis have increased in the United States. Kidney Int; 2013;83(3): 479-86.

2. Furyk JS, Chu K, Banks C, Greenslade J, Keijzers $\mathrm{G}$, Thom O, et al. Distal ureteric stones and tamsulosin: A double-blind, placebo-controlled, randomized, multicenter trial. Ann Emerg MedJan; 2016;67(1):86-95.

3. Bader MJ, Eisner B, Porpiglia F, Preminger GM, HG $\mathrm{T}$. Contemporary management of ureteral stones. Eur UrolApr; 2012;61(4):764-72.

4. Cui $X$, Ji F, Yan H, Ou TW, Jia CS, He XZ, et al. Comparison between extracorporeal shock wave lithotripsy and ureteroscopic lithotripsy for treating large proximal ureteral stones: a meta-analysis. UrologyApr; 2015;85(4):748-56.

5. Razzaghi MR, Razi A, Mazloomfard MM Golmohammadi TA, Valipour R, Safety RZ. and Efficacy of pneumatic lithotripters versus holmium laser in management of ureteral calculi: a randomized clinical trial. Urol J. 2013;10(1):76-26.

6. Akdeniz E, İrkılata L, Demirel HC, Saylık A, Bolat MS ŞN. A comparison of efficacies of holmium YAG laser, and pneumatic lithotripsy in the endoscopic treatment of ureteral stones. Turk J Urol. 2014;40(3):463-4.

7. Etafy M, Morsi GAM, Beshir MSM, Soliman SS, Galal HA OC. Management of lower ureteric stones: a prospective study. Eur J Urol. 2013;66(4):463-4.

8. Verze P, Imbimbo C, Cancelmo G, Creta M, Palmieri A MF. Extracorporeal shockwave lithotripsy vs ureteroscopy as first-line therapy for patients with single, distal ureteric stones: a prospective randomized studyNo Title. BJU. 2010;106(11):1748-52.

9. Kelleher JP, Plail RO, Dave SM. Sequential renography in acute urinary tract obstruction due to stone disease. Br J Urol. 1991 Feb. 67(2):125-8.

10. Segura JW, Preminger GM, Assimos DG Dretler SP, Kahn RI, Lingeman JE, al Ureteral Stones Clin Guidel Panel Summ Rep Manag ureteral Calc. 1997:158-65.

11. Knoll T, Alken P, Ms M. Progress in Management of Ureteric Stones. EAU Updat Ser. 2005;3:44-50.

12. Miller OF, Cj K. Time to stone passage for observed ureteral calculi: a guide for patient education. J Urol. 1999;162:688-90. 
13. LinksTiselius HG, Ackermann D, Alken P, Buck C, Conort P, on urolithiasis GM: G. No Title. Eur Urol. 2001;40:362-71.

14. Peschel R, Janetschek G, Bartsch G. Extracorporeal shock wave lithotripsy versus ureteroscopy for distal ureteral calculi: a prospective randomized study. J Urol. 1999:162-66.

15. Izamin I, Aniza I, Rizal AM, SM. A. Comparing extracorporeal shock wave lithotripsy and ureteroscopy for treatment of proximal ureteral calculi: a cost-effectiveness study. Med J Malaysia. 2009;64:12-15.

16. Stewart GD, Bariol S V, Moussa SA, Smith G. Matched pair analysis of ureteroscopy vs. shock wave lithotripsy for the treatment of upper ureteral calculi. Int J Clin Pr. 2007;61:784-8.

17. Parker BD, Frederick RW, Reilly TP, Lowry PS, Efficiency and cost of treating proximal ureteral stones: shock wave lithotripsy versus ureteroscopy plus holmium:yttrium-aluminium-garnet laser. Urology. 2004;64:110-2.

18. Kadyan B, Sabale V, Mane D, Satav V, Mulay A, Thakur $\mathrm{N}$, et al. Large proximal ureteral stones: Ideal treatment modality? Urol Ann. 2016;8:189-92.

19. Dickstein RJ, Kreshover JE, Babayan RK, DS. W. Is a safety wire necessary during routine flexible ureteroscopy? J Endourol. 2010;24:158-9.

20. Kira M, Atkin MS, Biri H, Ureteroscopy DN. the firstline treatment for distally located ureteral stones smaller than $10 \mathrm{~mm}$. Urol J. 2014;10:28-34.

21. Manzoor S, Hashmi AH, Sohail MA, Mahar F, Bhatti S. Extracorporeal shock wave lithotripsy (ESWL) vs. ureterorenoscopic (URS) manipulation in proximal ureteral stone. J Coll Physicians Surg Pak. 2013;23:26-30.

22. Islam M, Malik A. Ureteroscopic pneumatic versus extracorporeal shock wave lithotripsy for lower ureteral stones. J Coll Physicians Surg Pak. 2012;22:44-7.

23. Librenjak D. Eitum M, Gugic D, Milostic K, Duvnjak M. Ureterorenoscopic Treat ureteral stonesinfluence Oper Exp Ski Proced outcome. 2011;52:55-60.

24. Galal EM, Anwar AZ, El-Bab TK. Retrospective comparative study of rigid and flexible ureteroscopy for treatment of proximal ureteral stones. Int Braz J Urol. 2016;42:967-72.

25. Aboutaleb H, Omar M, Salem S, Elshazly M. Management of upper ureteral stones exceeding 15 $\mathrm{mm}$ in diameter: Shock wave lithotripsy versus semirigid ureteroscopy with holmium:yttriumaluminum-garnet laser lithotripsy. SAGE Open Med. 2016;4(20503):12116685180.

26. Lee YH, Tsai JY, Jiaan BP, Wu T, CC. Y. Prospective randomized trial comparing shock wave lithotripsy and ureteroscopic lithotripsy for management of large upper third ureteral stones. Urology. 2006;67:480-4.

27. Argyropoulos AN, Swl TDA. is more cost-effective than ureteroscopy and Holmium:Yag laser lithotripsy for ureteral stones: A comparative analysis for a tertiary referral centre. Br J Med Surg Urol. 2010;3:65-71.

28. Cho KS, Jung HD, Ham WS, Chung DY, Kang YJ, Jang WS, et al. Optimal Skin-to-Stone Distance Is a Positive Predictor for Successful Outcomes in Upper Ureter Calculi following Extracorporeal Shock Wave Lithotripsy: A \{B\}ayesian Model
Averaging Approach. PLoS One. 2015;10.

29. Pareek G, Armenakas NA, Panagopoulos G Bruno JJ, JA. F. Extracorporeal shock wave lithotripsy success based on body mass index and Hounsfield units. Urology. 2005;65:33-6.

30. Massoud AM, Abdelbary AM, Al-Dessoukey AA, Moussa AS, Zayed AS, Mahmmoud O. The success of extracorporeal shock-wave lithotripsy based on the stone-attenuation value from non-contrast computed tomography. Arab J Urol. 2014;12:155-61.

31. Alameddine M, Azab MM, ureteroscopy NAAS. Proximal versus distal ureteral stones. Urol Ann. 2016;8:84-6.

32. Al-Marhoon MS, Shareef O, Al-Habsi IS, Al Balushi AS, Mathew J, KP. V. Extracorporeal Shock-wave Lithotripsy Success Rate and Complications: Initial Experience at Sultan Qaboos University Hospital. Oman Med J. 2013;28:255-9.

DATA SHARING STATEMENT: The data that support the findings of this study are available on request from the corresponding author. The data are not publicly available due to privacy or ethical restrictions.

CONFLICT OF INTEREST: Authors declared no conflict of interest.

GRANTED SUPPORT AND FINANCIAL DISCLOSURE: Nil

AUTHOR'S CONTRIBUTION

Following authors have made substantial contributions to the manuscript as under

Ahmad S:

Concept and design of study, Collection of data, statistical analysis

Jamshed:

Shah A, Khan RA: Writing of manuscript, critical review of manuscript Analysis and interpretation of data, statistical analysis

Kalim M, Ali S: Data collection, bibliography

Authors agree to be accountable for all aspects of the work in ensuring that questions related to the accuracy or integrity of any part of the work are appropriately investigated and resolved. 